\title{
Addressing the Influencing Factors for Interoperability in a Global Domain
}

\author{
Tanmaya Kumar Das ${ }^{1}$, Dillip Kumar Mahapatra ${ }^{2}$ \\ ${ }^{1}$ Computer Science \& Engineering, C.V. Raman College of Engineering, India \\ ${ }^{2}$ Information Technology, Krupajal Engineering College, India
}

\begin{abstract}
Achieving interoperability in global context is difficult. Although the benefits of enabling systems to interoperate globally are significant, repeated failures to build working systems provide evidence that the tasks necessary to gain those benefits are poorly understood. Many organizations have addressed interoperability as primarily a technical issue [2]. However, to address the entirety of the interoperability challenge, development teams must also consider nontechnical factors that influence their efforts to meet interoperability goals. This report describes a proposed model through which one can understand interoperability in global context. With this model, system developers should characterize interoperability in six dimensions: Developers need to analyze interoperability requirements at the technical, semantic, and organizational levels, but they should also consider the legal, political, and socio-cultural issues with the system must also interoperate [5]. This paper explains some of the challenges associated with achieving interoperability in systems and presents some guidance on how to address interoperability requirements, with the goal of making both policy makers and system developers aware of the depth and breadth of enabling interoperability to provide many benefits, including improved efficiency, transparency, accountability, and access, as well as coordination of services at lower costs . However, repeated failures to build working systems show that the task is not only difficult but also poorly understood.
\end{abstract}

This report describes a proposed model for understanding interoperability in e-governance system context. With this model, system developers should characterize interoperability in six dimensions: technical, semantic, and organizational, as well as legal, political, and socio-cultural. This report also presents guidance on how to address interoperability requirements and describes challenges that policy makers and system developers face in achieving systems interoperability.

Keywords: Interoperability, Framework, Process agreement, Open standards, Catalogues.

\section{Introduction}

The term e-government is broadly defined as the use of information and communication technologies to support the business of government, such as providing or enhancing public services or managing internal government operations. Its benefits include improved efficiency, transparency, accountability, and access as well as coordination of services at lower costs. However, the task of providing these benefits are not only difficult but also poorly understood [5]. This work suggests that interoperability is a fundamental barrier to achieving the benefits of e-government. While many governments have addressed interoperability as primarily a technical issue and the interoperability problem has other facets and is influenced by a variety of sources, especially in the public service context. To address the entirety of the interoperability challenge [13], here it is essential to consider technical factors such as data semantics and process standardization as well as nontechnical factors such as legal, political, and social issues. This paper attempts to explain the challenges associated with achieving interoperability in e-government systems in order to provide a better understanding in context to its use and modifications whenever required.

\section{The Interoperability Problem}

Interoperability is a complex problem. To enable interoperability in a global context, all of the elements of e-government need to be examined.

\subsection{What is an Interoperability?}

The term Interoperability can be viewed in a wide range of possible meanings: the ability of systems to work together" in general or to be specific i.e. the ability of a set of communicating entities to (1) exchange specified geographical data and (2) operate on that geographical data according to specified, agreed-upon, operational semantics". Significant research has provided new ways to understand interoperability for many important stakeholders such as the computing community (significantly, the Institute of Electrical and Electronics Engineers), software research institutions [IEEE 1990]. System designers typically separate areas of concern (such as technical and nontechnical, as discussed in Section 4) by interoperability types and levels and 
organize them into interoperability models that present an overall perspective of interoperability in a given context.

\subsection{Models for Interoperability}

There are multiple models for interoperability. The models break down the interoperability problem into different types, levels, and/or dimensions. The Levels of Information Systems Interoperability (LISI) model breaks down interoperability into different levels of connectivity between systems [C4ISR Architectures Working Group 1998]. The Organizational Interoperability Maturity Model (OIMM) and the Levels of Conceptual Interoperability Model (LCIM) build on the LISI model in different ways - the OIMM model through abstraction to command-and-control support and the LCIM model through data management - to bridge technical design and conceptual design [4].

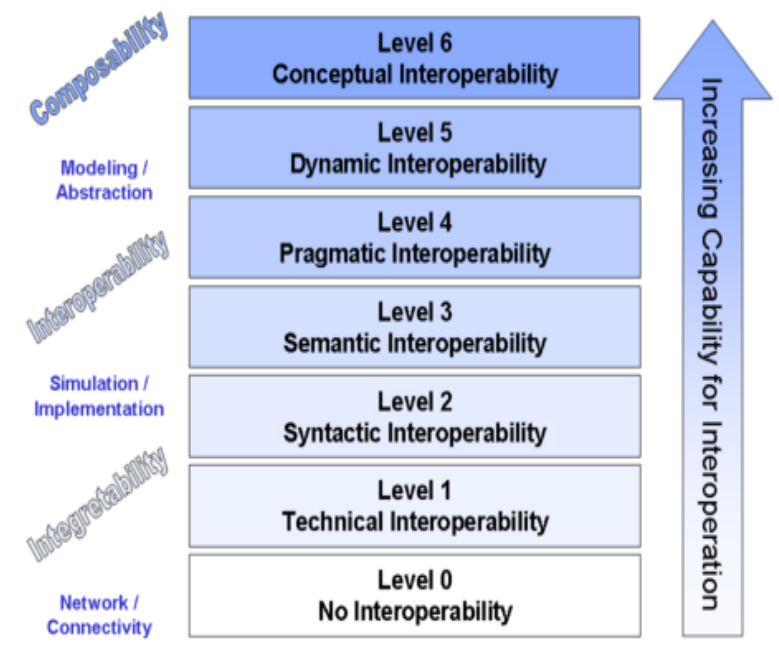

Figure 1: Conceptual Interoperability Model (LCIM)

\begin{tabular}{|l|l|}
\hline \multicolumn{1}{|c|}{ Levels } & \multicolumn{1}{|c|}{ Description of Interoperability at this level } \\
\hline L6 (Conceptual) & $\begin{array}{l}\text { Interoperating systems at this level are completely aware of each others information, processes, } \\
\text { contexts, and modeling assumptions. }\end{array}$ \\
\hline L5 (Dynamic) & $\begin{array}{l}\text { Interoperating systems are able to re-orient information production and consumption based on } \\
\text { understood changes to meaning, due to changing context as time increases. }\end{array}$ \\
\hline L4 (Pragmatic) & $\begin{array}{l}\text { Interoperating systems will be aware of the context (system states and processes) and meaning of } \\
\text { information being exchanged. }\end{array}$ \\
\hline L3 (Semantic) & Interoperating systems are exchanging a set of terms that they can semantically parse. \\
\hline L2 (Syntactic) & $\begin{array}{l}\text { Have an agreed protocol to exchange the right forms of data in the right order, but the meaning of data } \\
\text { elements is not established. }\end{array}$ \\
\hline L1 (Technical) & Have technical connection(s) and can exchange data between systems \\
\hline & \\
\hline
\end{tabular}

In general, developers define these models in terms of goals, types, and levels of interoperability. $\square$ An interoperability goal refers to a communication capability of a given system. For example, the most basic goal of interoperability is the exchange of information. The goals in a given model may range from the most basic, such as the exchange of information, to very complex, such as harmonized strategies in the LCIM [14]. The goals can also become more specific, depending on the granularity of the goals or the close relation to a particular domain of interest, which tends to result in specific goals related to that domain. $\square$ A type of interoperability usually specifies a domain of interest (such as network interoperability) or a goal within a specific interoperability model. For example, protocol interoperability is a specific and domain-dependent type of interoperability proposed within the Coalition Interoperability model that pertains to the goal that the communication protocols used on a network to support the necessary data exchange for the system $[7,8]$.

$\square$ Many models of interoperability present levels or layers of interqperability. As in any layered model, each goal or type of interoperability within the model is complementary and builds on one another in a stacklike form. In other words, the model presents a base goal or type of interoperability and then places all of the 
other goals or types on top in an order that specifies that each goal or type requires all of the goals of the levels below to be met to achieve its goal. Despite the similarities in how they are defined and structured, many of these models are unsuitable for defining a general interoperability model because of their domain-specific nature.

\section{A Proposed Model For Understanding Interoperability In The E-Government Context}

To understand interoperability in a general, domain-agnostic way, we propose a model that starts from the basic goals of interoperability and mapping these goals to levels, with the more complex goals mapped to higher levels of interoperability. Finally, we add the e-government context to the model as factors of influence. For clarity, we define a communication to be some exchange of data between two participants through some medium that may or may not have meaning attached. We define participants as the ultimate senders and receivers of the data exchanged, that is, the entities that use the data in a manner other than simply facilitating the exchange [13]. For example, in a scenario in which two humans communicate using cell phones, the humans are considered the participants and all of the hardware and software infrastructure elements facilitating the exchange are not considered participants as they only receive, process, and send the data to move it from one participant to the other. However, in such a system there are likely computer systems that monitor the conversation for audit logging, and thus the infrastructure may pass along direct or ancillary elements of the data exchange to these systems. Such systems, which use the data exchanged for purposes other than facilitating the communication, could also be considered participants, although whether or not they are considered active participants depends on the given context.

\subsection{Interoperability Goals}

There are three primary goals associated with achieving interoperability in any system(computer or otherwise): data exchange, meaning exchange, and process agreement.

\subsubsection{Data Exchange}

The first goal with respect to interoperability is basic data exchange i.e. whether data can be exchanged at all, May be data exchange range from phone connections, email, and document exchanges to web pages and the automated exchange of data in computer-readable format. A computer system example would be the exchange of data between two computer systems in which there is an agreement on the types and size of the data exchanged, and data can go back and forth without the participants having any knowledge of the meaning of the data.

\subsubsection{Meaning Exchange}

The second goal with respect to interoperability is the exchange of meaning (i.e., all participants in a given communication assign the same meaning to the information that is exchanged). Meaning exchange is fundamentally different from data exchange because of the aspect of misinterpretation. Data exchange either occurs or does not occur. Meaning exchange, however, is much more difficult because there is no implicit guarantee that all participants will interpret the meaning of the data in the same way. Even when two participants agree on a particular piece of data as a unit of distance, if both sides do not understand the specific type of unit in exactly the same way, there is potential for failure or even disaster.

\subsubsection{Process Agreement}

The third goal with respect to interoperability is agreement on how to act on information that has been exchanged (i.e., whether all participants in a given communication have the same understanding of how to act once they have exchanged information). Process agreement is a fundamentally different type of interoperability goal from data exchange and meaning exchange, because its focus shifts from the information exchanged to the actions taken by the participants once the information exchange has occurred. Here, all participants must agree in advance about what to do with the data they receive in the exchange. Process agreements are often complex and represent many of the problems that e-government efforts attempt to address. Lack of process agreement often manifests as a need for the consumer to provide the same information to multiple government services in response to a single event.

\subsection{Interoperability Levels}

The interoperability levels explain how interoperability goals can be built on each other to achieve various goals. On the basis of the interoperability goals presented in the previous section, we have established the following levels, as shown in Figure 1. 


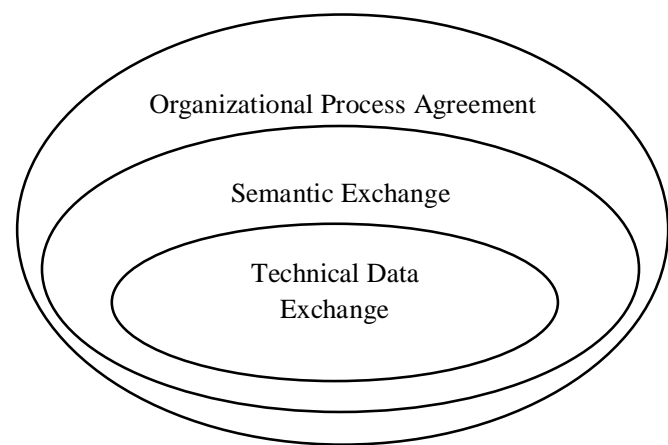

- $\quad$ Organizational Process Agreement : Participating in multi-organizational processes

- $\quad$ Semantic Exchange: Exchange of meaningful data across systems

- $\quad$ Technical Data Exchange :Exchange of technical data

Figure 2: Interoperability Levels

\subsubsection{Technical Interoperability}

Technical interoperability maps to the goal of data exchange. Here, technical interoperability is placed at the base level because the exchange of data is at the root of all communication. In some of the more technically based interoperability models, this level is divided into sublevels that map to specific modes of communication and separate the data from the communication channel [16]. The approach taken in existing egovernment interoperability models is to abstract the details of the communication and have a single level, which is the approach that is taken into in this paper.

\subsubsection{Semantic Interoperability}

Semantic interoperability maps to the goal of meaning exchange. It is placed above the technical interoperability level because in order to exchange meaning it is necessary to have already been successful at information exchange. This is consistent with many of the existing interoperability models [19].

\subsubsection{Organizational Interoperability}

Organizational interoperability maps to the goal of process agreement. We place it at the top level because process agreement cannot occur without both information exchange and meaning exchange supporting the communication to establish the process and the communication that contains the information for the recipient to act on. Although the term process interoperability describes the interoperability goal of process agreement more directly, organizational interoperability is a better term for the e-government context because it captures the scope of inter- and intra-organizational process alignment that is necessary to meet this interoperability goal.

\subsection{Influencing Factors}

The e-government context is complex because it has to deal with legal, politics and policy, and sociocultural issues. For example, the independent nature of government agencies and conflicting leadership, policy, and financial priorities make it difficult to enable consolidation and cooperation in the pursuit of better citizen services [12].

In this proposed interoperability model, we represent this additional impact of legal, political (policy), and socio-cultural issues as influencing factors. These factors are captured in this way and not as an additional level for these reasons:

- The impact of each is different, depending on the situation.

- $\quad \square$ They apply to almost any e-government system, regardless of the intended interoperability level.

For example, even a simple website that shares information with the public has to consider legal issues such as what information is considered public, political issues such as consistency with current policies and objectives, and socio-cultural issues such as accessibility for people using other languages for communication. From a system-developer perspective, these factors become an additional, critical dimension of the interoperability problem, and any e-government effort must address them. Reaching any interoperability level requires solutions to be compliant with legal, political, and socio-cultural factors, as shown in the proposed interoperability model in Figure 2. 

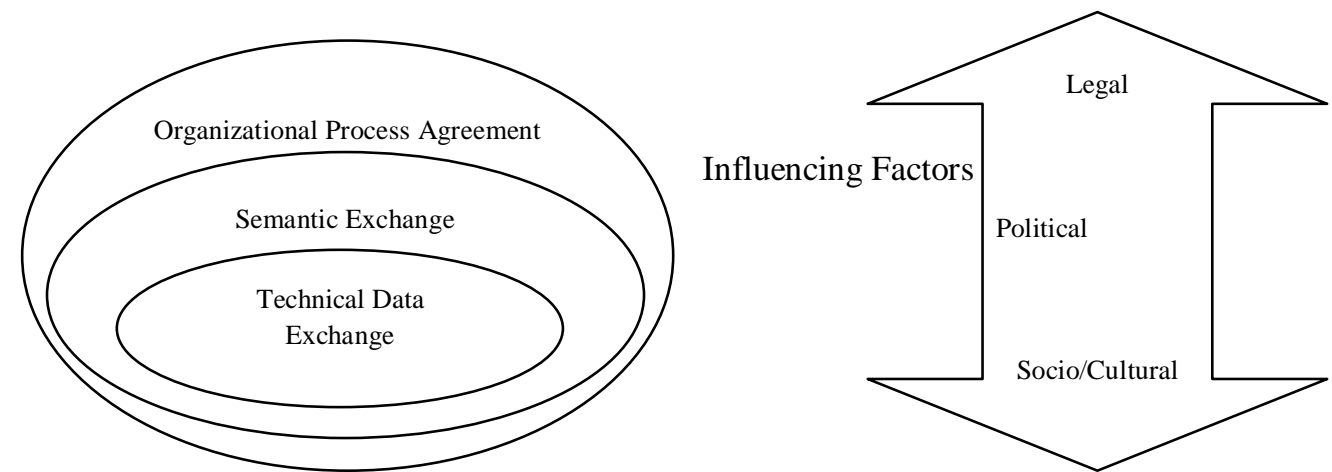

* Legal power assigned to system capabilities

*Political context and support for system capabilities

*Socio and cultural characteristics of system stakeholders

Figure 3: e-Government Interoperability Model

\subsubsection{Legal Factors}

One of the primary concerns from a legal standpoint is the assignment of legal weight to the outputs of a given e-government system so that the system can support or replace existing manual public services . Identifying legal issues inherent to public services is critical not only to enable appropriate e-government services but also to identify services that are not appropriate for the e-government context. The e-government systems must be legally responsible for these concerns, and this places a significant burden on the design, development, and maintenance of these systems. System designers must arrange for legal review of any potential failure of an e-government system to comply with laws and policies; determine the appropriate response to the failure; and, if necessary, carry out that response.

\subsubsection{Political Factors}

The political context in which we introduce e-government systems is critically important to the success of any e-government interoperability effort. For example, the general areas of concern that contain barriers to egovernment interoperability: policy makers, administrations, IT departments, and accessibility. Within those four areas, only two note any technical issues, while coordination among different agencies and departments is a common theme across all areas. A government administration that has the political will and power to organize, manage, and fund an e-government interoperability project in a way that addresses all of the interrelated issues will have a much better chance for success. Without this critical element, it can be difficult to realize the level of cooperation and coordination among the participating government departments necessary to address the technical, semantic, and organizational interoperability challenges that an e-government project may have.

\section{Addressing The E-Government Interoperability Problem}

The context for every e-government project is different: different services require different technologies, different semantics, and different processes, and they are influenced by different factors. The combination of desired services and the context in which the provider will deliver them drives interoperability requirements. Although we can apply concepts such as maturity levels or frameworks to the e-government context, this can be misleading [18] while frameworks and models can certainly help us set expectations and focus on problems.

\subsection{Interoperability Is Contextual}

To illustrate how different systems have different interoperability requirements, such as public services in countries with poor infrastructures, public services in developed countries and public services in highly connected countries. In case of poor infrastructure having limited computer and Internet access may have few community e-centers provide facilities that allow local residents to access basic services such as the Internet, and distance-learning tools as well as support other government services in the education, business, and health areas. Here, political factors come into play with the budgeting and management of the distribution effort.

In more developed countries, some government services are offered via web portals. To facilitate this, the website has to be implemented to capture information from the user, validate the information, perform the necessary actions, and inform the user of the results. This represents the technical interoperability requirements of the system. The website specifies how to enter certain information and provides additional help to fill out the fields on the form. This is an example of semantic interoperability requirements to make sure that the user understands the meaning of the entered information. To achieve this, the website has to be implemented to 
capture information from the user, validate the information, perform the necessary actions, and inform the user of the results. This represents the technical interoperability requirements of the system $[17,20]$. Highly connected countries, especially, provide examples of public services with high levels of interoperability. For example, if we use the case of electronic voting, we can assume there are technical interoperability requirements to confirm that a voter's vote is registered, semantic interoperability requirements to make sure that the voter voted for the desired candidate, and organizational interoperability requirements to carry out the collection, protection, counting, and certification of the votes. The legal, political, and social influencing factors for such a service should be clear and would cause the service to fail if designers did not address them (e.g., accessibility to ensure that legal citizens can vote, party-neutral user interface that does not show any preference or privileged position for any party, and audit mechanisms to support recounts or challenges to election results).

\subsection{Addressing Interoperability Requirements}

As all e-government systems are context dependent, every project must identify and analyze its interoperability requirements and the influencing factors relevant to its own context. Some guidance on interoperability requirements and influencing factors are addressed here.

\subsubsection{Addressing Technical Interoperability}

To enable information exchange at any level, governments must select compatible technology solutions. This can be as simple as standardizing a form or restricting communication to phone calls or as complex as selecting web service security standards. Regardless of the selected technology solution, service designers must consider a number of issues to ensure that the technology solution is appropriate. This effort should begin by examining how e-government stakeholders have historically attempted to solve the problem and identifying the issues relevant to their context.

\subsubsection{Technology Catalogs}

Here, establishing "standards catalogues" that contain approved technologies for use in a country's egovernment (may differ from one country to other). These catalogs enable a country to establish some commonality in the technologies used by different services across different agencies or ministries. Using technical standards to constrain technology procurement across departments and agencies is a proven method for promoting technical interoperability among large numbers of interacting entities [14].

\subsubsection{Use of Open Standards}

A common theme in the e-government context is the use of open standards to promote interoperability. Open standards are important to governments because they can increase the number of compatible offerings (which leads to lower switching costs) and because wide stakeholder participation may increase the quality and suitability of the standard. However, open standards are not always the solution because there will always be domains where open standards do not yet exist. So in some areas, at least for an interim period, governments may find themselves using so-called de facto standards that are controlled by a single company or small group of companies. In addition, the open and consensus based process that is typical of an open standard may not keep up with the rapid speed of innovation in the technology world. In the end, standards are successful if widely implemented, and wide implementation in turn depends on a number of factors that include the relevance of the standard, the process of adoption (open, transparent), and the quality of the documentation .

\subsubsection{Limitations of Standards}

Standards are clearly an important component of technical interoperability. However, we must stress the risks of placing too much emphasis on technical standards and the limitations of what a standards catalog will provide in the context of e-GIFs. First, the selection of technology standards will not guarantee semantic or organizational interoperability [14]. Even though there are languages for modeling information meaning and processes to support interoperation in these areas, the fundamental limitation is human agreement: All parties must agree on what a data point means and what to do with it. No current technology can make those decisions automatically, and it is unlikely that any technology ever will.

Second, there are risks in relying on standards to achieve technical interoperability. According to Lewis and colleagues, standards evolve, often break backward compatibility, are inconsistently specified, can become unstable or irrelevant, and can conflict or compete with other standards [14]. Also, mandating standards that are not yet mature enough for wide adoption can affect their evolution [CS Transform 2009, p. 3]. It must be noted that placing too much focus on standard selection can distract attention from the equally important effort of addressing semantic and organizational interoperability. Third, selecting a standard to solve a technical interoperability problem requires agreement, or at least rough consensus, and as a result it may not be possible 
for governments prospectively to list standards for all domains of interaction among the government, citizens, and businesses. This is especially true in nascent areas of technology, such as education:

Much of the technical content in many Interoperability frameworks is at a level of detail which, nowadays, is unnecessary. The market has matured significantly in recent years, so the solutions to many of what were previously seen as technical barriers to interoperability are now "designed in" to a wide choice of competitive, commercial products.

Technology changes over time and precipitates corresponding changes in any standards selected for an e-government effort. Thus, standards will need to be updated even if they are appropriate for the context at the time they are implemented. To fully leverage a technology standard, adopting organizations must actively manage the impact of changes to the standard on the systems in which it is used, which involves deciding when to adopt, upgrade, switch, and/or discontinue use. Failing to compensate for changes in the standard can result in a number of potential issues, such as incompatibilities with newer products and the need for work-arounds so that existing products retain their value.

\subsubsection{Addressing Semantic Interoperability}

To support semantic interoperability in a technological context, public service providers have to agree on several characteristics of the data to be exchanged, including data attributes such as:

- Units (e.g., metric versus imperial units)

- Validity (e.g., retirement-related information is valid only if the age of the person is greater than predefined criteria)

- Time period (e.g., a policy may not apply if an event occurred during a certain period of time)

Achieving semantic interoperability is a difficult problem that is largely unsolved within the e-government context. Stakeholders typically achieve semantic interoperability through direct negotiation until they reach a consensus. One common approach to building this consensus is to construct an ontology or set of ontologies, which are basically data models that define the data items that are exchanged - including the exact meaning and structure assigned to them - and the relationships between data items [4]. There are limitations in the use of ontologies and other semantic interoperability solutions. First, while data models generally cover only a single domain or area of concern, the typical e-government system will probably need to understand multiple areas of concern to operate correctly. For example, an emergency response system must be concerned about map data, weather data, logistics data, health data, and citizen data. Each of these areas of concern may represent the same data in a different way. Second, consumers of data models require different levels of detail, aggregation, and/or summarization. For example, a data model for meteorological data can be extremely detailed to support a scientist conducting studies on weather patterns and effects, whereas the typical consumer may be interested only in today's weather forecast. Third, areas of concern tend to evolve, requiring maintenance of existing data models and in some cases generation of new ones. Finally, it can be difficult for any community of interest to reach consensus on any area because people tend to interpret information in slightly different ways.

\subsubsection{Addressing Organizational Interoperability}

To enable organizational interoperability in the e-government context, public service providers have to agree on not only what information is exchanged, when it is exchanged, and how it is exchanged but also what to do with it when it is exchanged. Stakeholders typically achieve organizational interoperability in a manner similar to semantic interoperability, by negotiating directly to come to a consensus on the processes they will use. Addressing organizational interoperability is also similar to addressing semantic interoperability: The process is primarily manual, and a few tools are available to automate the process. Processes evolve in ways similar to data models and technological standards and thus incur the same maintenance costs. Agreement by communities of interest on processes can also be hard to reach for the same reasons and also because different organizations tend to use different business processes to create competitive advantages. Different organizations also have different (and sometimes directly conflicting) goals, so there may be scenarios in which technical and semantic interoperability exists between two government entities, but because of their competing goals the organizations will not agree to work together. Even though necessary for successful implementation of egovernment systems, dealing with legal, political, and socio-cultural influencing factors are excluded in this paper.

\section{Conclusion}

Systems interoperability is contextual. A corollary to this statement is that interoperability is not a characteristic of a single system but rather a characteristic of the relationship between two or more systems in a particular context. The context in which systems have to interoperate shapes the requirements that each individual system has to satisfy in order to interoperate with other systems. Thus, developers need to plan 
system components around both technical and nontechnical aspects of interoperability. A development team can analyze interoperability in any context along technical, semantic, and organizational dimensions, but the diversity and complexity of the legal, political, and socio-cultural dimensions of the e-government context make interoperability challenging for e-government systems. The model proposed in this report captures the differences between technical, semantic, and organizational interoperability while highlighting the crosscutting impact of legal, political, and socio-cultural issues as influencing factors. The goal of this model is to reinforce that developers need to characterize interoperability in the e-government context along the six dimensions of the model so that they can understand, discuss, and analyze it. If e-government system designers better understand the problem of interoperability and account for context-dependent influencing factors, we predict that more egovernment efforts will succeed.

\section{References}

[1] Wikipedia. Interoperability. http://en.wikipedia.org/wiki/Interoperability (Apr. 26, 2011).

[2] Biddick, Michael ."Government IT Priorities," InformationWeek Government. http://www.informationweek.com news /government/ enterprise-architecture/218500752 (July 18,2009).

[3] Wikipedia. Semantic Interoperability. http://en.wikipedia.org/wiki/Semantic_interoperability (Feb. 23, 2010).

[4] Wikipedia. Ontology Engineering. http://en.wikipedia.org/wiki/Ontology_engineering (Feb. 19, 2010).

[5] Brownsword, L., Carney, D. J., Fisher, D., Lewis, G., Morris, E. J., Place, R. R., Smith, J., Wrage, L., \& Meyers, B. C. Current Perspectives on Interoperability (CMU/SEI-2004-TR-009). Software Engineering Institute, Carnegie Mellon University, 2004.

[6] C4ISR Architectures Working Group. Levels of Information Systems Interoperability. U.S. DoD, OSD (C3I), CIO, Director for Architecture and Interoperability, 1998.

[7] Clark, T. \& Jones, R. "Organisational Interoperability Maturity Model for C2." Proceedings of the 1999 Command and Control Research and Technology Symposium. Newport, RI, June 1999. CASOS, 1999.

[8] CS Transform. Beyond Interoperability: A New Policy Framework for e-Government. CS Transform, 2009.

[9] E-Government for Development Information Exchange. What Is eGovernment? University of Manchester's Institute for Development Policy and Management, 2008.http://www.egov4dev.org/success/definitions.shtml

[10] Global Standards Collaboration. "Resolution GSC-14/24: (IPRWG) Open Standards (Reaffirmed). "Presented at the 14th Global Standards Collaboration meeting, July 2009.http://www.itu.int/dms_pub/itu-t/oth/21/05/T2105000011002400MSWE.doc

[11] Government of India. Policy on Open Standards for E-Governance. Ministry of Communications \& Information Technology, 2008. http://egovstandards.gov.in/policy/policy-on-open-standardsforegovernance/policy_doc_and_manual_used_in_printing_recd_on_Nov_12.pdf/at_download/file

[12] Biddick, Michael. "Government IT Priorities," InformationWeek Government.

[13] http://www.informationweek.com/news/government/enterprise-architecture/218500752 (July 18,2009).

[14] Lallana, Emmanuel C. e-Government Interoperability. United Nations Development Programme,2008.

[15] Lewis, Grace, Morris, Edwin, Simanta, Soumya, \& Wrage, Lutz. "Why Standards Are Not Enough to Guarantee End-to-End Interoperability," 164-173. Proceedings of the Seventh International Conference on Composition-Based Software Systems (ICCBSS 2008). Madrid, Spain, Feb. 2008. IEEE Computer Society Press, 2008.

[16] OMG. Business Process Model and Notation (Version 2.0) (OMG Doc. No. formal/2011-01-03). Object Management Group, 2011. http://www.omg.org/spec/BPMN/2.0/PDF

[17] The Open Group. Welcome to TOGAF-The Open Group Architecture Framework. http://www.opengroup.org/architecture/togaf8doc/arch/toc.html (1999-2008).

[18] Open ePolicy Group. Roadmap for Open ICT Ecosystems. Berkman Center for Internet \& Society at Harvard Law School, 2005.

[19] Organization for the Advancement of Structured Information Standards. Web Services Business Process Execution Language Version 2.0. OASIS, 2007. http://docs.oasisopen. org/wsbpel/2.0/wsbpel-v2.0.pdf

[20] Pardo, Theresa A. \& Burke, G. Brian. Improving Government Interoperability: A Capability Framework for Government Managers. Center for Technology in Government, SUNY Albany,2008.

[21] Pyarelal, Suchitra. "Interoperability and Open Standards for e-Governance." e-Gov (Sep. 1, 2007):17-19. 